\title{
THE CREATION OF QUANTUM CHROMODYNAMICS
}

Gerardus 't Hooft

Institute for Theoretical Physics, Utrecht University, The Netherlands 


\title{
THE CREATION OF QUANTUM CHROMODYNAMICS
}

\author{
Gerardus 't Hooft \\ Institute for Theoretical Physics, Utrecht University \\ Princetonplein 5, 3584 CC Utrecht, The Netherlands \\ email: g.thooft@fys.ruu.nl
}

April 3, 1998

\begin{abstract}
This is a personal account of those events which, in the eyes of the author, were the most crucial ones in the development of our insights in the nature of the strong interactions.

\section{PREHISTORY}

In the early ' $60 \mathrm{~s}$, most physicists did not believe that strong interactions were described by a conventional quantum field theory. Much effort went into the attempts to axiomise the doctrine of relativistic quantum particles strongly interacting among themselves. All we had, for sure, was that there should exist an $S$-matrix, obeying all sorts of requirements: unitarity, causality, crossing symmetry [1] [2].
\end{abstract}

One then turned to the experimental evidence that strong interactions have a well-documented symmetry structure. There is a practically exact $U(2)$ 
symmetry (isospin and baryon number conservation), a softly broken $S U(3)$ (M. Gell-Mann's "Eightfold way" [3]), and a more delicate chiral $S U(2) \times S U(2)$ that is both spontaneously broken (so that the pions became massless Goldstone bosons) and explicitly broken (the pions still have a non-vanishing mass). Although the possibility of a substructure for the strongly interacting particles — the quark theory [4] — was soon realised, it was nevertheless suspected that not the quarks but the Noether currents associated with the symmetries were playing an essential role in the dynamics. It was attempted to write down rigorous algebras for these currents, such that their representations would generate the sought-for $S$-matrix [5]. They were combined with unitarity, causality and crossing symmetry as these were suggested by quantum field theory, but one did not want to use quantum field theory itself because of the infinities generated by the inevitable renormalization procedure [6].

A promising new idea was "duality" (in terms of the Mandelstam variables $s, t$ and $u$ for elastic scattering events) [7]. A great discovery by G. Veneziano [8] was that one could write down simple mathematical expressions for amplitudes that obeyed this duality requirement. Since this duality is somewhat different from what one has in standard quantum field theory, it was clear why there was much enthusiasm for it. After Z. Koba and H.-B. Nielsen found how to generalise Veneziano's expressions to $n$-particle amplitudes [9], the physical interpretation was also discovered, by Nielsen [10], T. Goto, L. Susskind and Y. Nambu [11]: the amplitudes are the scattering amplitudes for strings colliding, merging and splitting one against the other.

However, these string theories were confronted with difficulties concerning unitarity, which appeared to require the presence of tachyons. The latter are physically not observed, and furthermore would lead to technical 
inconsistencies in the theory. Later, it was found how to safeguard string theories against this disease, but then they would produce a gravitational force, and require supersymmetry. This made these theories more suitable for Planck length physics, but it disqualified them for the "ordinary" strong interactions. We now know that the "string theory for the strong interactions" is only approximately true; in QCD, color fields tend to form vortices that behave similarly to strings, and this explains the early qualitative successes of the dual models.

What were the alternatives? In 1960, Gell-Mann and M. Lévy [12] had constructed a simple renormalizable model for the strong interactions, the socalled 'sigma-model' 1 , in which the nucleons, $p$ and $n$, and the pions, $\pi^{ \pm}$and $\pi^{0}$ played a dominant role, together with a new hypothetical particle, the $\sigma$. In this theory, chiral $S U(2) \times S U(2)$ was spontaneously and explicitly broken much in the way actually observed, and some of the features that had been derived from the algebra of the chiral currents could be explicitly demonstrated in this model, which made it very attractive. This produced, in 1969, a short revival of quantum field theory. It was extensively studied by B.W. Lee [13], J.-L. Gervais [14] and K. Symanzik [15] $]^{2}$. Yet, the model contained one very large coupling constant, the pion-nucleon coupling constant $g \approx 13$, which made it clear that ordinary perturbation expansions make no sense.

There were hopes that perturbation expansion could be improved. The

1 In mathematical physics, sigma models are popular subjects of investigation; the origin of the name is usually forgotten.

2 These authors were certainly aware of the fact that the explicit chiral symmetry breaking could be attributed to the mass terms of the $u$ - and the $d$-quark, even if the details of the quark dynamics were not understood. 
magic word ${ }^{3}$ was 'Padé approximation', a rearrangement of the perturbative corrections in terms of rational functions of the perturbation parameter $g$. Successes were reported in obtaining phenomenologically reasonable expressions, but it became clear that this procedure could not serve as a theoretically sound framework.

Meanwhile, experiments were moving towards the higher energy domain, and here a peculiar scaling phenomenon was observed, and the general nature of this behaviour was investigated by J.D. Bjorken [17]. This experimental discovery became to be known as "Bjorken scaling". It was subsequently interpreted by Feynman [18] as if, at short distances, constituent particles inside hadrons move comparatively freely. He called these constituents 'partons'. It would later turn out to be a good insight not to call them 'quarks', because now we know that partons can be both quarks and gluons.

The study of scaling behaviour of renormalizable theories became a central point around 1970. Independently, in the late '60s, C.G. Callan [19] and K. Symanzik [20] found equations obeyed by the renormalized Green functions that follow from their scaling behaviour. By inspecting the Lagrangian of a field theory, and the ensuing classical field equations, one might have expected that the behaviour of such theories under scale transformations is quite simple, especially in cases where one can ignore the effects from the particle mass terms. But these authors found that the quantum corrections to scaling behaviour are actually quite subtle: the naive scaling equations are violated by the radiative corrections. It should be stressed that these radiative corrections depend on many details of the model under consideration, and Callan and

3 The need for "magic" is a deplorable weakness in any theory [16]. 
Symanzik only studied Quantum Electrodynamics (an Abelian gauge theory) and theories involving scalar particles, not the non-Abelian gauge theories. Functions in their equations they called $\beta$-functions, describe how the effective coupling constants actually depend on the scale. This scale dependence had been found earlier by Gell-Mann and F. Low [21]. The fact that the redefinitions of the renormalized coupling constants form a group had been emphasized earlier by Peterman and Stueckelberg [22], hence the name 'renormalization group', but only the subgroup that corresponds to scale transformations has physically significant consequences.

Callan and Symanzik explicitly constructed their equations only for the theories mentioned earlier, but they expected them to have universal validity. In particular, the $\beta$-functions were found to be positively valued, and this too was thought to be a universal feature, valid for all quantum field theories, Abelian as well as non-Abelian ones. There were mathematical arguments supporting this view, arguments which were only later seen to be flawed.

In the late ' $60 \mathrm{~s}$, there were various developments in the domain of YangMills theories. Models for the electroweak interactions had been proposed by Glashow [23], Salam [24], and Weinberg [25], but they did not know how these models should be renormalized while avoiding the complications due to ghost particles with 'negative metric', which means that these violate unitarity. Consequently, these investigators turned their attention elsewhere.

After some pioneering work by R.P. Feynman [26] and B.S. DeWitt [27], the investigation of pure Yang-Mills theories was pursued by a group in Leningrad (now St. Petersburg), L. Faddeev and V. Popov [28], and in Moscow, E.S. Fradkin and I.V. Tyutin [29], although these authors were primarily interested in the closely related, but much more difficult problem of quantizing gravity. Yang-Mills gauge theory with a mass term explicitly added 
(without Higgs mechanism) had been introduced by Feynman [26] and Glashow [23], and this avenue was pursued by Veltman [30].

In their studies of the Gell-Mann-Lévy $\sigma$-model, it was observed, by B.W. Lee and Symanzik, that renormalization and symmetry breakdown went well together. As a student at that time, I was interested in applying this observation to what is now called the Higgs mechanism. The Higgs mechanism differs in several important respects from spontaneous breakdown of a global symmetry. Firstly, the Higgs effect alters in an essential way the particle spectrum, as it removes scalar particle modes while adding the physical transverse helicity states of the gauge vector particles. Secondly, it adds various kinds of "ghost particles" to the Feynman rules of the system. Thirdly, the implications of local gauge invariance were ill understood, and the exact prescriptions for higher loop diagrams were unknown. Veltman had pointed out to me that scalar particles can only exacerbate the unitarity problems of massive vector particles, if you try to use them to remove the $k_{\mu} k_{v}$ terms in the vector propagator. Yet this was when you used derivative couplings, instead of applying gauge invariance. My suspicion was that nevertheless the Higgs mechanism should be essential for renormalization, and it appeared to allow for a treatment quite similar to that of the $\sigma$-model within perturbation theory. Discussions with Lee and Symanzik were not helpful at that time, as they both confessed not to know much about Yang-Mills theories. My advisor Veltman did not want to hear about fields with vacuum expectation values.

Realizing that the world was not yet ready to accept the renormalizable Higgs theories, I returned to the case of pure, massless Yang-Mills fields. At that time, renormalization was generally thought to be a trick that happened to work only in very special cases such as Quantum Electrodynamics. In 1970, the details of the renormalization procedure for pure Non-Abelian gauge theories 
were worked out [31]. In this paper, a new set of extended Ward identities was derived, and their essential significance for the renormalizability was proved ${ }^{4}$.

Generalization towards gauge theories with Higgs mechanism was now straightforward. A key to the understanding of the renormalizability of theories with massive gauge bosons was the realization that mass terms should never be inserted by hand, but exclusively evoked by a Higgs mechanism. I now could convince Veltman of this fact, by providing the exact Feynman rules and the Ward identities needed for renormalization [34]. These renormalization procedures were immediately extended to all Abelian and non-Abelian gauge theories, with or without Higgs mechanism $[34]^{5}$. Numerous investigations were carried out to further streamline the renormalization procedures, among which the introduction of dimensional regularization and renormalization [35] and BRST quantization [33].

Only after the difficulties of the massless and massive Yang-Mills theories were cleared, did theoreticians have the tools in their hands to construct theories such as Quantum Chromodynamics.

\section{AWAKENING}

In the '60s, various attempts were made to construct Yang-Mills theories for the strong interactions. Models were invented in which the vector mesons

4 These identities were later to be called Slavnov-Taylor identities [32], because these authors extended the identities to off-shell amplitudes. The only reason why the offshell expressions were not written down earlier was that they create new infinities, requiring the renormalization of wave functions and composite operators, while these were not directly needed for the renormalization of the Yang-Mills fields (for the massless as well as the Higgs case). The identities were later understood to be a consequence of an induced anticommutative symmetry by Becchi, Rouet and Stora, and by Tyutin [33].

5 Provided that some chiral anomalies are made to cancel out. 
were treated as Yang-Mills bosons, with a mass term added to the Yang-Mills action. This was not a bad idea, because it explained a phenomenon called vector meson dominance, an experimentally observed feature of the electromagnetic form factors of the nucleon [36]. Of course, at that time, it was not known how to renormalize these models. We now know that they are not renormalizable ${ }^{6}$.

What was really needed, however, was a model that described quarks. Ever since Gell-Mann had launched the 'Eightfold Way', the idea that hadrons are made up of quark-like constituents [4] was kept alive. The big question was how these quarks are bound together. Gell-Mann knew about Yang-Mills theory. H. Fritzsch, who had just escaped from the DDR, joined him in his research; he too had studied Yang-Mills theory. They emphasized that, if you attach a color degree of freedom to quarks, you remove an apparent contradiction arising from the Fermi-Dirac statistics of quarks on the one hand, and the observed $S U(3)$ symmetric representation spectrum of baryons on the other [37]. All that was needed now was a confining two-body or three-body force that selects out color singlet states for the hadrons, rendering all color-nonsinglets unphysical. Exactly why they are unphysical (do such states carry infinite energy, or is it enough to say that they are not gauge-invariant?) was unclear [38].

The notion of color as an internal symmetry for quarks had been introduced by O.W. Greenberg [39], and it was used by M.Y. Han and Nambu [40] in their model for the strong interactions. They avoided the quark confinement problem by invoking a mixing mechanism for color and

6 They could be cured, by invoking Higgs mechanisms, but these effective strong interaction theories suffer from other severe defects as well. 
electromagnetism in such a way that quarks receive integral charges. This idea is not as far off from the truth as one might think. If, in modern QCD, scalar fields would exist in the fundamental representation of $S U(3)$, regardless whether they possess a vacuum expectation value or not ${ }^{7}$, one would be able to treat these as Higgs fields, to represent the situation in the way Han and Nambu did [41].

Gell-Mann, Fritzsch and H. Leutwyler proposed an unbroken $S U(3)$ Yang-Mills theory for quarks [38]. It was not clear to them whether or to what extent one had to modify the Yang-Mills potential in order to achieve quark confinement and Bjorken scaling. They thought of non-local terms in the action, but they also suggested that complicated dynamical mechanisms could exist that take care of the problem.

In spite of their beauty, these ideas were taken with skepticism. The explanation of quark confinement was not considered to be adequate. This skepticism encouraged a new series of experiments at CERN, where the highest energy $p p$ collider, ISR, allowed to check if quarks remained confined at these energies [42]. It seemed to be a miracle that quarks can move freely at high energies and yet they stick together inseparably inside the hadrons. To many physicists, Bjorken scaling appeared to be a signal that no quantum field theory would be fit to account for the observed strong interactions [43]. Why then did nobody compute the $\beta$-function for Yang-Mills theories? I had computed this function, and knew about its sign. In 1971, as soon as we knew how to renormalize the theory, I started a preliminary calculation, showing the negative sign. Not realizing that this was something totally novel, I could not imagine

7 This would not be a gauge-invariant statement anyway. 
that this was all that was needed to resolve the outstanding problems of strong interaction theory. I actually thought that this scaling behaviour was also known, but not considered relevant for partons. Since I could not understand their problems, I turned away from the strong interactions that had to be infinitely complicated.

By 1972, when Veltman and I had worked out many examples of gauge models, we knew how to compute the renormalization counter terms, and I had found a way to relate these to the equations governing the running coupling constants. Veltman was not interested in these findings. According to him, they were only relevant for Euclidean space, which has nothing to do with experimental observations, all done in Minkowski space. On several occasions I mentioned to him the possibility of a pure gauge theory for the strong interactions, but his mind was made up. As long as they produced no answer to the quark confinement problem, such ideas are useless, and no referee would accept a publication suggesting such an idea.

In June 1972, a small meeting was organised in Marseille by C.P. Korthals Altes. Symanzik was there. Upon arrival, right at the airport, Symanzik and I began to discuss our work. Symanzik explained that he worked on $\lambda \phi^{4}$ theory with negative $\lambda$, because of its interesting scaling properties [44]. He had been attracted by the idea that theories with a negative $\beta$-function would reproduce parton-like behaviour, but, the only models with such properties that he could think of were theories with a negative squared coupling constant, $g^{2}<0$ or $\lambda<0$.

Naturally, I asked him how he would solve the problem that the energy appears to be unbounded from below. He explained that perhaps this problem could be cured by non-perturbative effects, which at that time were very badly understood. But I informed him of my finding that the coefficient determining 
the running of the coupling strength, that he called $\beta\left(g^{2}\right)$, for gauge theories is negative. Symanzik was surprised and skeptical. "If this is true, it will be very important, and you should publish this result quickly, and if you won't, somebody else will," he said.

In the meeting, Symanzik ended his talk on negative $\lambda \phi^{4}$ theories by the remark that further study on the $\beta$-functions for non-Abelian gauge theories were called for. When it was time for questions I came forward to write down on the blackboard the expression I had derived:

$$
\frac{\mu d g^{2}}{d \mu}=\frac{g^{4}}{8 \pi^{2}}\left(\frac{-11}{3} C_{1}+\frac{2}{3} C_{2}+\frac{1}{6} C_{3}\right)
$$

for a theory in which the gauge group has a Casimir operator $C_{1}$ and where fermions have a Casimir operator $C_{2}$. Scalars contribute with the Casimir operator $C_{3}$. In the $S U(2)$ case this would mean that with less than 11 fermions in the elementary representation (and no scalars) the $\beta$-function would be negative, and for $S U(3)$ the critical number would be $33 / 2$.

Why did I not follow Symanzik's sensible advice to publish this result? Weinberg had written a long paper in which he reported about the complicated infrared structure of gauge theories, which made me believe he knew everything about what was to be called 'infrared slavery', a direct consequence of 'asymptotic freedom'. Surely, I thought, he understands the scaling behaviour of gauge theories. David Gross had explained to me that Bjorken scaling cannot be accommodated for in any field theory, Abelian or not. Veltman was telling me that scaling all components of the momentum vector would lead you far away from the mass shell, whereas all experiments were looking at on-massshell hadronic final states, so the deep-Euclidean region was unphysical and 
uninteresting ${ }^{8}$. As for my views about the small-distance structure of quantum field theories, I felt like crying in a desert. If I would write a paper claiming that a pure $S U(3)$ Yang-Mills theory could explain the strong interactions, it would be directly dismissed. I was made to believe that the real question was not the scaling, but the understanding of quark confinement. I felt that, as long as I could not argue why quarks are confined, nobody would listen to these ideas. Veltman convinced me that our work on gravity was more important, and it kept us quite busy.

As for what exactly happened in the States, I can only repeat the accounts given by others [43] [45]. D. Gross and F. Wilczek at Princeton set out to prove that no quantum field theory could ever yield a negative $\beta$-function, so that Bjorken scaling would require a radical departure from quantum field theory. H.D. Politzer at Harvard was given the problem as a student by his advisor S. Coleman. Both groups obtained an answer almost simultaneously, but when they compared what they had, there was an embarassment: Politzer had found a negative $\beta$, whereas the Princeton group had what they expected, a positive $\beta$. But they soon found their sign error, and the correct result was quickly agreed upon. Two papers were published in the same issue of Physical Review Letters [46], and these were the first published accounts of the negative beta function. Politzer suggested that his finding would render the non-Abelian perturbation expansion 'reliable'; this was perhaps a bit too optimistic, but, of course, perturbative approaches would indeed come within reach at the highenergy domain. It was realized that Bjorken scaling would still be violated by

8 I should have known that this view was not at all shared by everyone. Amplitudes with exchanges at high, spacelike values for $q^{2}$ are certainly physically relevant; indeed, very high spacelike $q^{2}$ are now directly experimentally accessible. 
logarithms, but the general feeling was that the logarithms would be accounted for, as indeed they would.

When I heard about these papers, I was surprised, not about the result, which I had known all along, but about the stir they caused. And, finally, people began to talk about a pure $S U(3)$ theory for quarks. Well, I thought, they still have nothing, as Veltman had taught me, what really matters is the quark confinement problem. No real advances had been made yet. I set out to try to understand it from first principles. QCD had only come half-way.

The original calculations of the QCD $\beta$-functions were rather complicated, as was the calculation which I had presented in Marseille. In the meantime, however, a much more elegant and faster procedure had been invented, since it was observed that these $\beta$-functions are closely related to the counter terms in the dimensional regularization scheme [47], and then the calculation of these counter terms was streamlined by using background field methods [48]. The original $\beta$-functions used by Callan and Symanzik had a complicated mass dependence in them. This, however, was not essential. If one uses different definitions of the coupling constant, one can eliminate this mass dependence. The dimensional renormalization procedure could be associated with what was later called 'minimal subtraction': the pure subtraction of only the dimensional poles from the amplitudes. The $\beta$-functions then become mass-independent. The importance of this result is that the picture of what is going on physically is significantly simplified. One can read off the $\beta$-functions quickly from the Lagrangian of the theory. Only in the case that particles have a very large mass, do the expressions develop new large logarithms, which can be removed by considering the heavy particles to be 'decoupled', but then the coupling constants have to be redefined, and the $\beta$-functions change. 


\section{CONFINEMENT}

Several key developments then helped us to understand confinement. The string-interpretation of the dual models gave a first clue: mesons behave as little stretches of strings, with quarks at their end points. This most beautifully squared with the observed linear relation between the mass-squared and the angular momentum of the heavy mesonic resonances, the so-called Regge trajectories ${ }^{9}$. This now suggests that the colored forces between quarks form vortex lines that connect color charges. How can one understand why these are formed? This is a long-distance problem, and precisely at these long distances (of a Fermi and more), the coupling strength grows so large that perturbative arguments will be meaningless.

Independently, Nielsen and P. Oleson [51] in Copenhagen, and B. Zumino [52] at CERN, discovered a new and extremely important feature in an Abelian Higgs theory: this model would allow for the existence of a magnetic vortex line that would have several features in common with the dual string: it is unbreakable, its mass-energy content is entirely due to the tension force, and it exhibits an internal Lorentz invariance (for boosts in the direction of the string). If quarks would be magnetic monopoles with respect to this spontaneously broken $U(1)$ force, you would get exactly the model you want. In fact, this model is nearly identical to much older models for superconductor material in which the Meissner effect is active. The vortices are just the wellknown Abrikosov vortices formed by magnetic fields that succeed in penetrating into a superconductor.

But quarks are not monopoles. This theory was not QCD, and so it would not explain Bjorken scaling and the symmetric $S U(3)$ representations of the

9 The work of J. Chew [1], S. Frautschi [49] and V. Gribov [50] should be mentioned here. 
hadrons. What happens if one replaces the Abelian $U(1)$ theory by a nonAbelian theory with Higgs mechanism? Does that also allow for Abrikosov vortices? I asked myself this question, and ran into a puzzle: there would be vortices, but they would differ from the Abelian ones in that now they can break. The resolution of the puzzle was revolutionary: the vortices break because they can create pairs of magnetic monopoles and antimonopoles. It was not known yet that magnetic monopoles can exist in fairly ordinary non-Abelian gauge models! I had now learned not to sit upon such a result for long. The paper was published in which I explained the nature of the magnetic monopoles in gauge theories [53]. Independently, this discovery was made in Moscow: A. Polyakov had constructed the same gauge field solution [54], although he did not immediately realize that the object carries a single magnetic charge.

Magnetic monopoles turned out to be a magnificent laboratory for the study of the remarkable topological features of gauge theories. When I was back at Utrecht, Peter Hasenfratz was a young postdoc there, and he asked me questions that made me realize the existence of a very peculiar feature in gauge theories with monopoles. We wrote a paper about it together [55]. Imagine a gauge theory without any fermions, but there are (bosonic) fields with gaugeisospin of $1 / 2$. If the Higgs field carries isospin 1, we have magnetic monopoles with magnetic charge $2 \pi / e$ and we have electrically .charged particles with charge $e$. Both will be bosons with integral spin. However, if these two form a bound state, it will have orbital angular momentum that is halfinteger [55]. Moreover, I can give beautiful arguments (but never published) that this bound state made of only bosons actually obeys Dirac-Fermi statistics. $^{10}$ I thought it was great to get fermions out of the blue, in a theory

10 Other arguments for this, such as the one published by A. Goldhaber [56], appear to be much more indirect and difficult to follow. 
that was initially without fermions.

However, it looked as if the monopoles was a side-track. By itself it did not bring us closer to understanding quark confinement; yet it would later turn out to be a crucial ingredient, as I will explain shortly.

First, something else happened. K. Wilson was studying the renormalization group for non-Abelian gauge theories restricted on a space-time lattice. His interest was primarily in the domain of computer simulations. But he made a crucial observation [57]: if, for practical, technical reasons, you restrict (Euclidean ${ }^{11}$ ) space and time to form a lattice, you can perform a new perturbative expansion: the $1 / g^{2}$ expansion. This large coupling constant expansion becomes accurate at large distance scales. Surprisingly, one can read off right away that the only states that one can then talk about are gaugeinvariant (that is, colorless) states, formed by quarks that had to be connected to each other by line segments of the lattice, exactly as in the dual string theory! In other words, quark confinement is evident and absolute in the large coupling constant expansion!

In my recollection, this was the first indication that quark confinement is absolute, quarks can never escape from each other. It looks as if, for a theory on a lattice, when the coupling constant is varied from small to large, a phase transition occurs. QCD is said to be in the confinement phase. Yet, in the continuum theory, the interactions are generated at very tiny distance scales, where the coupling constant is weak, and then, by cumulative effects, they generate larger couplings at large distances. Does this justify a large coupling constant expansion for continuum theories? Not really. We were coming closer, but the exact nature of the confinement mechanism had not yet been

11 And, of course, by this time, the relevance of Euclidean space for the physical aspects of the theory was obvious. 
explained convincingly.

In statistical physics, there is a famous exactly solvable model, called the Ising Model (fïst solved by L. Onsager [58]). The model has a dual symmetry that relates small coupling (high temperature) to strong coupling (low temperature). A phase transition occurs at the so-called duality point [59]. In a super conductor, the phase transition to superconductivity is of a comparable nature. In QCD, we have color electric fields and color magnetic fields. If there were a Higgs mechanism, it would be the magnetic fields that form vortex lines, much like the ones Nielsen, Olesen and Zumino had derived in a $U(1)$ theory with Higgs mechanism. In addition, as Polyakov and I had shown, there would be free magnetic monopoles. They would be at the end points of the magnetic vortices. The confinement mechanism that we wanted to understand, would require the color-electric field lines to form stringlike vortices. The colorelectric quarks should be at the end points. Clearly, the relevant duality symmetry here is electric-magnetic duality.

Because of Dirac's relation [60] between the fundamental electric charge quantum $e$ and the magnetic charge quantum $g_{m}$,

$$
g_{m} e=2 \pi n \hbar c
$$

where $n$ is an integer, the interchange electric-magnetic also corresponds to a small coupling-large coupling interchange, just as in the Ising model. Therefore, to understand confinement, one would have to interchange the electrically charged particles with the magnetic monopoles in the theory. If, subsequently, a Higgs mechanism is assumed to take place among the magnetic monopoles, we obtain the dual analogue of the older model of Nielsen, Olesen and Zumino. The magnetic vortex in that model would now manifest itself as an electric 
vortex line, and the role of the monopoles in that model would now be played by the quarks. They will be held together absolutely and permanently by these vortices. Bingo, this is permanent quark confinement.

I had the opportunity to report this mechanism in my rapporteur's talk [61] at the Palermo Conference of 1975. S. Mandelstam had the same idea [62]. The next step would be to reformulate this mechanism in more precise terms. This was done by carefully rephrasing the gauge constraints of the nonAbelian theory. It had been noted by Gribov [63], that the usual procedure for fixing the gauge degree of freedom in an Abelian gauge theory leads to a complication when applied to the non-Abelian case: the Lorenz condition does not fix the gauge freedom completely; there is an ambiguity left. This ambiguity is harmless as long as one sticks to a perturbative expansion. There, we know exactly what kind of non-local effects are caused by gauge fixing: they are the Faddeev-Popov ghosts. The procedure of treating them is wellunderstood in perturbation expansion.

But in attempts to go beyond perturbation expansion, Gribov's ambiguity is important. It is then important to be able to distinguish physical states from ghosts. Ghosts arise as soon as the gauge fixing procedure requires the solution of differental equations; their kernels are the ghost propagators. If one wants to avoid ghosts altogether, one must use a local, non-propagating gauge fixing procedure. Indeed, it is possible exactly to give a gauge constraint that removes the non-Abelian parts of the local gauge invariance, without any ambiguity [64]. After such a gauge fixing procedure, one is left with an apparently Abelian effective theory, with in addition to the electrically charged particles, magnetic monopoles. They emerge as singularities due to the gauge fixing. Since the Abelian gauge group that survives is the Cartan subgroup of the original gauge group (the largest Abelian subgroup), we call this procedure the 'maximal 
Abelian gauge fixing procedure', or 'the Abelian projection'.

In the maximal Abelian gauge, we find 'electrically' charged particles, 'magnetically' charged particles, each with finite masses depending on the dynamical features of the system, and massless $U(1)$ 'photons', that couple to the 'electric' and 'magnetic' particles in a totally symmetric fashion. Of course, these 'electric' and 'magnetic' charges are not the charges that couple to the ordinary photons. We should call them 'color-electric' and 'color-magnetic', and the $U(1)$ photons they couple to are really gluons. All one is now left with to do, is to invoke the Higgs mechanism in this color regime. If the Higgs mechanism applies to the color-electrically charged particles, then they will roam around freely, only interacting with each other at short ranges, since they exchange gluon-photons that now have become massive. The color-magnetic particles will be held together eternally by color-magnetic vortex lines. But the effective model in the maximal Abelian gauge equally well allows us to assume the Higgs mechanism to work on the color-magnetically charged particles, in which case the color-electrically charged particles are held together by colorelectric vortices, being the dual strings. Whether the color-electric, or the colormagnetic Higgs mechanism takes place, or perhaps neither, depends on the relative strength of the color-electric and the color-magnetic charges. Due to asymptotic freedom, one generally expects the color-electric charges to become large when it is attempted to separate them by large distances; the colormagnetic charges will become small, with a tendency to be screened completely. If one assumes that the latter undergo Bose condensation, just as in super conductors and super fluids, one has permanent quark confinement.

The above is not a proof that quarks in QCD are confined, but it is a description of the mechanism; whether it actually occurs depends on dynamical features such as the ratios of various forces in the theory, and in most cases this 
can only be established by detailed numerical analysis.

This analysis has now been done. A daring experiment was first carried out by M. Creutz et al, on a computer [65]: they took a lattice in fourdimensional Euclidean space and defined the QCD field variables on this lattice. Because of the complexity of the problem, and the limited capacity of these early computers, the lattices had to be very modest in size. Nevertheless, their computer simulations showed clear evidence of a transition towards a confining phase. Nowadays, by using much larger and faster computers, as well as much improved analytical procedures, this transition can be studied in much more detail. One has also been able to impose maximal Abelian gauge conditions on the leading color field configurations that were obtained, and quite generally, the picture of Bose-condensing magnetic monopoles has been confirmed [66]. Today, it is fair to say that quark confinement is no longer seen as a deep puzzle in quantum field theory. We therefore understand why protons did not break into quarks in the ISR experiments at CERN [42]. If a color vortex ever breaks, it is because of quark-antiquark pair formation, so that hadrons are always kept color neutral. A vortex breaking without quark pair formation would require large-scale structures (i.e., low energy excitations) of a kind that directly contradict the lattice simulations. Therefore, we do not expect that experimentalists will ever detect free quarks. If fractional electric charges will ever be observed, this will be highly interesting from a theoretical point of view, but these objects will have little to do with quarks.

\section{THE U(1) ANOMALY}

Permanent quark confinement was not the only puzzle that had to be solved before the more tenacious critics would accept QCD as being a realistic theory. There had been another cause for concern. This was the so-called $\eta-\eta^{\prime}$ 
problem. The problem was evident already to the earliest authors, Fritzsch, Gell-Mann and Leutwyler [38]. The QCD Lagrangian correctly reflects all known symmetries of the strong interactions:

- baryon conservation, symmetry group $U(1)$;

- isospin, symmetry group $S U(2)$, broken by electromagnetism, and also a small mass difference between the up and the down quark;

- an $S U(3)$ symmetry, broken more strongly by the strange quark mass;

- spontaneously broken chiral $S U(2) \times S U(2)$, also explicitly broken by the mass terms of the up and the down quark. The explicit chiral symmetry breaking is reflected in the small but non-vanishing value of the pion mass-squared, relative to the mass-squared of other hadrons;

- spontaneously broken chiral $S U(3) \times S U(3)$, explicitly broken down to $S U(2) \times S U(2)$ by the strange quark mass, which leads to a relatively light kaon.

Furthermore, the hadron spectrum is reasonably well explained by extending these symmetries to an approximate $U(6)$ symmetry spanned by the $d, u$ and $s$ quark, each of which may have their spins up or down.

The problem, however, is that the QCD Lagrangian also shows a symmetry that is known to be badly broken in the observed hadron spectrum: instead of chiral $S U(2) \times S U(2)$ symmetry (together with a single $U(1)$ for baryon number conservation), the Lagrangian has a chiral $U(2) \times U(2)$, and consequently, it suggests the presence of an additional axial $U(1)$ current that is approximately conserved. This should imply that a fourth pseudoscalar particle (the quantum numbers are those of the $\eta$ particle) should exist that is as light as the three pions. Yet the $\eta$ is considerably heavier: $549 \mathrm{MeV}$, instead of 
$135 \mathrm{MeV}$. For similar reasons, one should expect a ninth pseudoscalar boson that is as light as the kaons. The only candidate for this would be one of the heavy mesons, originally called the $X^{0}$ meson. When it was discovered that this meson can decay into two photons [67], and therefore had to be a pseudoscalar, it was renamed as $\eta^{\prime}$. However, this $\eta^{\prime}$ was known to be as heavy as $958 \mathrm{MeV}$, and therefore it appeared to be impossible to accommodate for the large chiral $U(1)$ symmetry breaking by modifying the Lagrangian. Also, the decay ratios - especially the radiative decays - of $\eta$ and $\eta^{\prime}$ appeared to be anomalous, in the sense that they did not obey theorems from chiral algebra [68].

A possible cure for this disease was also recognised: the Adler-BellJackiw anomaly. The divergence of the axial vector current is corrected by quantum effects:

$$
\partial_{\mu} f_{\mu}^{A}=\frac{g^{2}}{16 \pi^{2}} \varepsilon_{\mu \nu \alpha \beta} \operatorname{Tr} G_{\mu \nu} G_{\alpha \beta}
$$

where $J_{\mu}^{A}$ is the axial vector current, $G_{\mu \nu}$ the Yang-Mills gluon field, and $g$ the strong coupling constant. So, the axial current is not conserved. Then, what is the problem?

The problem was that, in turn, the r.h.s. of this anomaly equation can also be written as a divergence:

$$
\varepsilon_{\mu v \alpha \beta} \operatorname{Tr} G_{\mu \nu} G_{\alpha \beta}=\partial_{\mu} K_{\mu}
$$

where $K_{\mu}$ is the Chern-Simons current. $K_{\mu}$ is not gauge-invariant, but it appeared that the latter equation would be sufficient to render the $\eta$ particle as light as the pions. Why is the $\eta$ so heavy?

There were other, related problems with the $\eta$ and $\eta^{\prime}$ particles: their 
mixing. Whereas the direct experimental determination of the $\omega-\phi$ mixing [69] allowed to conclude that in the octet of vector mesons, $\omega$ and $\phi$ mix in accordance to their quark contents:

$$
\omega=\frac{1}{\sqrt{2}}(u \bar{u}+d \bar{d}), \quad \phi=s \bar{s},
$$

the $\eta$ particle is strongly mixed with the strange quarks, and $\eta^{\prime}$ is nearly an $S U(3)$ singlet:

$$
\eta \approx \frac{1}{\sqrt{3}}(u \bar{u}+d \bar{d}-s \bar{s}), \quad \eta^{\prime} \approx \frac{1}{\sqrt{6}}(u \bar{u}+d \bar{d}+2 s \bar{s}) .
$$

Whence this strong mixing?

Several authors came with possible cures. Kogut and Susskind [70] suggested that the resolution came from the quark confinement mechanism, and proposed a subtle procedure involving double poles in the gluon propagator. Weinberg [71] also suggested that, somehow, the would-be Goldstone boson should be considered as a ghost, cancelling other ghosts with opposite metrics. My own attitude [61] was that, since $K_{\mu}$ is not gauge-invariant, it does not obey the boundary conditions required to allow one to do partial integrations, so that it was illegal to deduce the presence of a light pseudoscalar.

Just as it was the case for the confinement problem, the resolution to this $U(1)$ problem was to be found in the very special topological structure of the non-Abelian forces. In 1975, a topologically non-trivial field configuration in four-dimensional Euclidean space was described by four Russians, A.A. Belavin, A.M. Polyakov, A.S. Schwarz and Yu.S. Tyupkin [72]. It was a localized configuration that featured a fixed value for the integral

$$
\int d^{4} x \varepsilon_{\mu \nu \alpha \beta} \operatorname{Tr} G_{\mu \nu} G_{\alpha \beta}=\frac{32 \pi^{2}}{g^{2}} .
$$


The importance of this finding is that, since the solution is localized, it obeys all physically reasonable boundary conditions, and yet the integral does not vanish. Therefore, the Chern-Simons current does not vanish at infinity. Certainly, this thing had to play a role in the violation of the $U(1)$ symmetry.

This field configuration, localized in space as well as in time, was to be called "instanton" later [73]. Instantons are sinks and sources for the chiral current. This should mean that chirally charged fermions are created and destroyed by instantons. How does this mechanism work?

Earlier, R. Jackiw and C. Rebbi [74] had found that the Dirac equation for fermions near a magnetic monopole shows anomalous zero-energy solutions, which implies that magnetic monopoles can be given fractional chiral quantum numbers. We now discovered that also near an instanton, the Dirac equation shows special solutions, which are fermionic modes with vanishing action [73]. This means that the contribution of fermions to the vacuum-to-vacuum amplitude turns this amplitude to zero! Only amplitudes in which the instanton creates or destroys chiral fermions are unequal to zero. The physical interpretation of this was elaborated further by Russian investigators, by Jackiw, Nohl and Rebbi [75], and by C. Callan, R. Dashen and D. Gross [76]: instantons are tunnelling events. Gauge field configurations tunnel into other configurations connected to the previous ones by topologically non-trivial gauge transformations. During this tunnelling process, one of the energy levels produced by the Dirac equation switches the sign of its energy. Thus, chiral fermions can pop up from the Dirac sea, or disappear into it. In a properly renormalized theory, the number of states in the Dirac sea is precisely defined, and adding or subtracting one state could imply the creation or destruction of an antiparticle. This is why the original Adler-Bell-Jackiw anomaly was first found to be the result of carefully renormalizing the theory. 
With these findings, effective field theories could be written down in such a way that the contributions from instantons could be taken into account as extra terms in the Lagrangian. These terms aptly produce the required mass terms for the $\eta$ and the $\eta^{\prime}$, although it should be admitted that quantitative agreement is difficult to come by; the calculations are exceptionally complex and involve the cancellation of many large and small numerical coefficients against each other. But it is generally agreed upon (with a few exceptions) that the $\eta$ and $\eta^{\prime}$ particles behave as they should in QCD ${ }^{12}$.

\section{REFINEMENTS}

Time has come to address more detailed questions. First, we would also like to understand why chiral $S U(2) \times S U(2)$ and chiral $S U(3) \times S U(3)$ are spontaneously broken. Banks and Susskind [79] came with a neat argument. In a pseudoscalar meson, the quarks are in an $S$-state, which means that they move primarily in the radial direction. In the color gauge field, the quark helicity should be conserved, so when the radial velocity switches sign, so should the quark spin. Such a simultaneous switch appears to be difficult to understand. It is more likely that the quarks simply continue their ways into the Dirac sea while other inhabitants of this sea take their place on the way back. This, these authors argue, implies that $q \bar{q}$ must have a large expectation value in the vacuum. This is spontaneous chiral symmetry breaking.

A second argument came from the effective Lagrangians for the hadrons. The terms due to instantons that represent the breakdown of chiral $U(1)$ conservation, also add to the effective potentials terms that induce spontaneous symmetry breaking. But a more convincing argument comes from a careful

12 There were protests. R. Crewther continued to disagree [77]. It was hard to convince him [78]. 
consideration of all possible Adler-Bell-Jackiw anomalies that might arise in the presence of "weak spectator gauge fields" (such as ordinary electroweak fields, but one may also imagine superweak interactions that have not yet actually been detected). We simply require that the effective theories reproduce the correct anomalies. This is called the anomaly matching condition [80]. It turns out to be extremely restrictive. Although it does allow chiral $S U(2) \times S U(2)$ to be in the Wigner mode, this is not the case [13] for chiral $S U(3) \times S U(3)$. If we assume that the small value of the strange quark mass has little effect on the vacuum expectation values of non-strange operators, chiral symmetry must be spontaneously broken.

Can we analyze QCD with more numerical precision, and formulate new predictions? Enormous amounts of work have been done. The simulations of QCD on a lattice are making good progress. On the one hand, computers are getting larger and faster. This helps, but not in a very spectacular way. The simulation programs must be improved, and this is also being done. The trick is to suppress two sources of error: one is the effects of the finiteness of the lattice size. So-called improved actions [81] are being designed that are being chosen in such a manner that the small-lattice-size limit converges as fast as is possible. Secondly, the effects due to the small volume of the total system should be suppressed: usually the size is chosen in such a way that the particle under consideration, say the proton, just barely fits in there. But we can try to predict the effects of the boundary, so that they can be corrected for. This, however, is usually considered to be very difficult.

Various different models have been proposed that are related to QCD proper, but have been modified in such a way that analytical calculations are possible. A first simplification is to replace the number $N$ of color degrees of freedom, which is 3 for the physical theory, by a number tending to infinity. In 
the $N \rightarrow \infty$ limit, mesons become non-interacting [82], and baryons behave as solitons (a proposal to treat baryons as solitons in a meson theory has been proposed long ago by T.H. Skyrme [83], and so these baryons are now called skyrmions). The coupling constant between the mesons is essentially $1 / N$. Thus, it was proposed to consider the $1 / N$ expansion. Unfortunately, the $N \rightarrow \infty$ limit is still an extremely complicated theory, and closed expressions for, say, the meson mass spectrum have not been found. It is tempting to suspect that some modified string theory should accommodate for them, but this is not known, in spite of numerous attempts and conjectures.

What can be solved exactly is the $N \rightarrow \infty$ limit in two dimensions (one space, one time dimension) [84]. It is comforting to observe that in this limit one not only sees confinement, with a calculable spectrum of linearly rising trajectories of mesonic resonances, but also chiral symmetry is seen to be broken spontaneously, with the pion mass-squared being linearly proportional to the up and the down quark masses, when these are small. Working out the Skyrmion spectrum in this model is much more difficult.

Recently, considerable progress was reported concerning supersymmetric versions of QCD [85]. In many supersymmetric models, the vacuum state can be characterized accurately, and many of our insights concerning the confinement mechanism, the role of instantons and chiral symmetry breaking could be verified explicitly [86].

\section{COMPARING WITH EXPERIMENT}

The experimental data that were used for the construction of QCD were the spectra of the lowest hadronic states, and the linearity of the so-called Regge trajectories (i.e. the linear relation between mass-squared and angular momentum for all mesonic and baryonic resonances). $\mathrm{QCD}$, having only one 
coupling parameter that fixes the scale of the interactions, and for the rest requiring only a few quark masses, explains these features remarkably well. But the truly new predictions are the perturbative expressions that should gain more and more accuracy at higher energies. The first spectacular event was the $J / \psi$ discovery. The exotic features of $J / \psi$ were not predicted ${ }^{13}$ but they should have been. Due to their large masses, the charmed quark and antiquark move in a region that is much smaller than that of all other mesons. Hence they probe QCD where its coupling is relatively small. The decay rate, of which the first stage leads to an intermediate state with three gluons,

$$
c \bar{c} \rightarrow g g g
$$

is governed by a factor $g^{6}$, where $g$ is the QCD coupling constant. Furthermore, the $c \bar{c}$ wave function tends to spread, yielding even more powers of $g$. So, if $g$ decreases, the decay rate drops very fast. This explains the small decay rate of $J / \psi$. But, since we can here compute the interquark potential and the wave functions, many of the details of the other bound states could be predicted. The story was repeated for the beauty quark, and, much more recently, for the top quark, for which the gauge coupling is even further reduced.

But it should be possible to check QCD more directly at very high energies. When quarks or leptons hit each other at small impact parameters, we get events where quarks and gluons are produced with large transverse momenta. A theory was developed that describes how these quarks and gluons evolve and turn into hadronic states. They form showers of hadrons, called jets

13 They nearly were, by Th. Appelquist and H.D. Politzer [87], but their paper about the $c \bar{c}$ bound state came after $J / \psi$ had been discovered. 
[88]. The first phases of this process can be handled by perturbation expansion, whereas the final phases must be universal for all types of jets. This way, the properties of the jets initiated by quarks and gluons could be predicted. If, during the initial phase, additional gluons are produced by radiative effects, multi-jet events will result. Apparently, however, theoreticians were unable to prescribe what experimentalists had to look for to establish the universal nature of these final interactions. The experimental results were discouraging; scattering experiments yielded different final states for each pair of interacting particles. So it happened that these aspects of QCD had to wait until experimentalists themselves came with the right idea [89]. The showers come with what is now called an "effective energy", and, in terms of this quantity, universality could be established [90].

On a statistical basis, one can now distinguish quark jets from gluon jets, and this enables us to check QCD predictions at high energies. Hadronic decay events of the $Z$ and $W$ bosons for instance, will be good laboratories, since in these cases the initial states are very precisely known. If the initial particles are nucleons, one has to insert information about the nucleonic wave functions in terms of quarks and gluons. The ratios of the occurence of sea quarks and valence quarks have been studied, but other aspects of the nucleonic wave function need further clarification.

When at very high energies, larger nuclei are collided one against the other, one expects an effective heating of hadronic matter. At sufficiently high temperatures, transitions may take place in which quarks get deconfined. Experimental signals for such a new plasmalike phase have been investigated. The importance of investigating the quark-gluon plasma lies in the fact that during the evolution of the early universe, matter may have been in this phase for a short time. 


\section{THE $\theta$ ANGLE}

In the absence of massless fermions, the color fields may freely perform transitions from one gauge configuration into a topologically rotated one. Since these gauge rotations are an exact invariance of the system, forming the (additive) group of integers, $\boldsymbol{Z}$, the vacuum state must be a representation of this gauge group, which is characterized by an angle $\theta$. If there are massless fermions, the effects of this angle disappear [73] [75] [76], while the angle itself merely fixes the direction in which chiral symmetry is explicitly broken. One can also say that, with respect to the axis specified by the $\theta$ angle, a fermionic mass term becomes a complex number:

$$
m \rightarrow m\left(\cos \theta+i \gamma^{5} \sin \theta\right)
$$

The imaginary part of this mass violates $\mathrm{CP}$ invariance, and so, $\mathrm{CP}$ invariance breaking is predicted by QCD, but the amount of breaking - being proportional to $\sin \theta-$ is unpredictable. Since explicit CP violation also occurs elsewhere in particle physics (notably the kaon system), the choice $\theta=0$ is not an option. Virtual kaons will renormalize $\theta$ into a value different from zero.

One of our problems is to determine $\theta$ from experimental observations. Strong CP breaking, however, has not (yet) been observed. This implies that $\theta$ must be very small.

The smallness of $\theta$ is puzzling. Is there a conspiracy that tunes $\theta$ towards zero? A popular proposal is that there is a spontaneous symmetry breaking effect acting on a new pseudoscalar field that is coupled to $\theta$, the socalled axion. The axion may be a relatively light, not strongly interacting pseudoscalar elementary particle. It has been searched for for a long time, without success.

The $\theta$ parameter cannot be tuned by an experimentalist; it is a God- 
given number. But if we could tune it, it would be interesting to calculate what might happen as $\theta$ varies. Theoretical computations have been made. Notably, one predicts one or more phase transitions as $\theta$ runs from zero to $2 \pi$. If nowhere else, then a phase transition must occur [64] [91] at $\theta=\pi$. At this point, the monopole field that causes confinement by undergoing the Higgs mechanism, must switch places with a monopole-gluon bound state.

$\theta$-dependence plays an important role in the supersymmetric models, where $\frac{1}{8 \pi^{2} g^{2}}+\theta i$ is taken to be the primary physical parameter.

Studying these $\theta$ effects will also be of importance because a repetition of the story is expected for the electroweak theory. Here, instanton-related effects will be excessively weak, except perhaps at very high energies and very high multiplicities of the final states. In this theory, instantons induce a symmetry breaking that is more spectacular than in the strong interaction theory: it is the baryon number itself that is destroyed. Still earlier in the evolution of the universe, when quarks and fermions, together with photons and intermediate vector bosons, formed an electroweak plasma [92], instantons were creating and destroying baryonic matter, by forming metastable intermediate solitonic particles, called 'sphalerons' [93]. The observed baryon excess in the present universe must be due to an asymmetry in these instanton related effects. This asymmetry can only be explained if we assume that the universe at this state was far away from equilibrium. Baryons probably were created in excess of antibaryons during the time that bubbles of true vacuum configurations appeared in a supercooled but symmetric pseudovacuum. The required asymmetry occurred in the rapidly expanding bubble walls [94].

\section{REMAINING QUESTIONS}

QCD is an extremely complex theory, in spite of the extreme simplicity of 
its basic equations. Both from the experimental side, and from the theoretical side, important issues have to be resolved. Some theoretical questions are extremely fundamental. To start with, it has not been proven that QCD is mathematically airtight. We would like to have prescriptions for computing hadronic states, or wave functions, for ordinary particles such as the pion and the proton, as well as scattering matrix amplitudes, which should be infinitely accurate, at least in principle. This is a hard question, considering the fact that even for the classical problem of $N$ gravitating particles such questions are not easy to answer. Next, we would like to possess decent approximation methods that convert reasonably rapidly to the desired numerical answers. Presently, we do have the Monte Carlo schemes on a lattice, but here convergence is painfully slow.

It is a big challenge to analyse in more detail the large $N$ expansion ( $N$ being the number of colors) [82]. As stated before, in the large $N$ limit, mesons are non-interacting, so it should be relatively easy to compute their spectrum. Unfortunately, this is not easy. One expects that freely moving strings, modified by stiffness terms in their Lagrangian, should describe these states, but this has not been shown. It is also worth-while to continue efforts to first put the $N \rightarrow \infty$ domain of QCD on a rigorous mathematical footing [95]. That would leave us with a $1 / N^{2}$ expansion, which, with $N=3$, may lead to results that can be checked experimentally.

Extremely difficult from the theoretical point of view, is the treatment of the many particle in, many particle out, scattering process. Even if the initial state is simple, like an electron-positron annihilation event, the many-particle final state is difficult to compute, or even estimate. Notably the $\theta$-dependent effects are difficult. This was dramatically exhibited in the electroweak theory, where, in spite of the smallness of the coupling constants, transitions into 
multiples of $1 / \alpha$ final particles, generated hot disputes as to whether the instanton effects will be negligible, or perhaps even dominant. The quark-gluon plasma is an extreme example of a multi-particle problem. It will have to be handled by statistical quantum field theory, a doctrine that has already matured.

Experimentalists should study the complete arena of ultra-high multiplicity events, in particular when a simple initial state, such as $e^{+} e^{-}$, gives rise to a very high multiplicity of low energy hadronic particles.

Lattice theorists should continue to produce faster algorithms, devise improved actions, and make models for the boundary effects of the (periodic) lattice walls, which hopefully will enable them to perform precision tests and comparisons with experiment.

\section{CONCLUSION}

Quantum Chromodynamics is a magnificent theory. Not one physicist, or even one group of physicists, can claim the discovery of this theory. Many new insights were needed to convince the scientific community that this set of equations, a pure $S U(3)$ Yang-Mills theory with a handful of massive fermions and essentially no scalars, can completely account for all observed strong interactions. It is now one of the best established branches of the Standard Model, but it may still keep theoreticians as well as experimentalists busy for quite some time to come. 


\section{REFERENCES}

[1] G. Chew, in The Analytic S-Matrix, W.A. Benjamin Inc. (1966).

[2] M. Goldberger, in The Quantum Theory of Fields, Proceedings of the 12th Solvay Conference, Interscience, New York (1961).

[3] M. Gell-Mann, "The Eightfold Way - A Theory of Strong-Interaction Symmetry", California Institute Technology Synchrotron Lab. Report 20 (1961); Y. Ne'eman, Nuclear Physics 26 (1961) 222; see also: M. Gell-Mann and Y. Ne'eman, "The Eightfold Way", Benjamin, New York (1964).

[4] M. Gell-Mann, Physics Letters 8 (1964) 214; G. Zweig, Erice Lecture 1964, in Symmetries in Elementary Particle Physics, A. Zichichi, Ed., Academic Press, New York, London, 1965, and CERN Report No. TH 401, 4R12 (1964), unpublished.

[5] M. Gell-Mann, Physics 1 (1964) 63; R. Dashen and M. Gell-Mann, Physical Review Letters 17 (1966) 340; S. Adler, Physical Review 140B (1965) 736; W.I. Weisberger, Physical Review 143B (1966) 1302.

[6] R.P. Feynman, in The Quantum Theory of Fields, Proceedings of the 12th Solvay Conference, Interscience, New York (1961). See also: T.Y. Cao and S.S. Schweber, The Conceptual Foundations and Philosophical Aspects of Renormalization Theory, Synthese 97 (1993) 33, Kluwer Academic Publishers, The Netherlands.

[7] V. Alessandrini, D. Amati, M. Le Bellac and D. Olive, Physics Reports $1 \mathrm{C}$ (1971) 269; J.H. Schwarz, Physics Reports 8C (1973) 270; S. Mandelstam, Physics Reports $\underline{13 \mathrm{C}}$ (1974) 259.

[8] G. Veneziano, Nuovo Cimento 57A (1968) 190.

[9] Z. Koba and H.B. Nielsen, Nuclear Physics B10 (1969) 633, ibid. B12 (1969) 517; B17 (1970) 206; Zeitschrift für Physik 229 (1969) 243.

[10] H.B. Nielsen, "An Almost Physical Interpretation of the Integrand of the npoint Veneziano model", XV Int. Conf. on High Energy Physics, Kiev, USSR, 1970; Nordita Report (1969), unpublished; See also: D.B. Fairlie and H.B. Nielsen, Nuclear Physics B20 (1970) 637. 
[11] H.B. Nielsen and L. Susskind, CERN preprint TH 1230 (1970), Y. Nambu, Proceedings International Conference on Symmetries and Quark models, Wayne State University (1969); Lectures at the Copenhagen Summer Symposium (1970); T. Goto, Progr. Theor. Phys. 46 (1971) 1560; L. Susskind, Nuovo Cimento 69A (1970) 457; Physical Review 1 (1970) 1182.

[12] M. Gell-Mann and M. Lévy, Nuovo Cimento $\underline{16}$ (1960) 705.

[13] B.W. Lee, "Chiral Dynamics", Gordon and Breach, New York (1972).

[14] J.-L. Gervais and B.W. Lee, Nuclear Physics B12 (1969) 627; J.-L. Gervais, Cargèse lectures, July 1970.

[15] K. Symanzik, Cargèse lectures, July 1970.

[16] G. 't Hooft, "The Limits of our Imagination in Elementary Particle Theory", to be published in 35th course, Highlights: 50 Years Later, Erice, Aug.Sept. 1997.

[17] J.D. Bjorken, Physical Review 179 (1969) 1547.

[18] R.P. Feynman, Physical Review Letters $2 \underline{3}$ (1969) 337.

[19] C.G. Callan, Physical Review D2 (1970) 1541.

[20] K. Symanzik, Commun. Math. Phys. 16 (1970) 48; ibid. 18 (1970) 227, ibid. $\underline{23}$ (1971) 49.

[21] M. Gell-Mann and F. Low, Physical Review 95 (1954) 1300.

[22] E.C.G. Stueckelberg and A. Peterman, Helv. Phys. Acta 26 (1953) 499; N.N. Bogoliubov and D.V. Shirkov, Introduction to the theory of quantized fields, Interscience, New York (1959); A. Peterman, Physics Reports 53C (1979) 157.

[23] S.L. Glashow, Nuclear Physics 22 (1961) 579.

[24] A. Salam and J.C. Ward, Physics Letters 13 (1964) 168, A. Salam, Nobel Symposium 1968, ed. N. Svartholm.

[25] S. Weinberg, Physical Review Letters 19 (1967) 1264.

[26] R.P. Feynman, Acta Phys. Polonica 24 (1963) 697. 
[27] B.S. DeWitt, Physical Review Letters 12 (1964) 742, id., Physical Review 160 (1967) 1113; ibid. 162 (1967) 1195, 1239.

[28] L.D. Faddeev and V.N. Popov, Physical Review 25B (1967) 29; L.D. Faddeev, Theor. and Math. Phys. 1 (1969) 3 (in Russian), Theor. and Math. Phys. 1 (1969) 1 (Engl. transl.).

[29] E.S. Fradkin and I.V. Tyutin, Physical Review D2 (1970) 2841.

[30] M. Veltman, Physica 29 (1963) 186, Nuclear Physics B7 (1968) 637; J. Reiff and M. Veltman, Nuclear Physics B13 (1969) 545; M. Veltman, Nuclear Physics B21 (1970) 288; H. van Dam and M. Veltman, Nuclear Physics B22 (1970) 397.

[31] G. 't Hooft, Nuclear Physics B33 (1971) 173.

[32] A. Slavnov, Theor. Math. Phys. 10 (1972) 153 (in Russian), Theor. Math. Phys. 10 (1972) 99 (Engl. Trans1.); J.C. Taylor, Nuclear Physics B33 (1971) 436.

[33] C. Becchi, A. Rouet and R. Stora, Commun. Math. Phys. 42 (1975) 127; id., Annals of Physics (N.Y.) 98 (1976) 287; I.V. Tyutin, Lebedev Prepr. FIAN 39 (1975), unpublished.

[34] G. 't Hooft, Nuclear Physics $\underline{\text { B35 }}$ (1971) 167.

[35] G. 't Hooft and M. Veltman, Nuclear Physics B44 (1972) 189; C.G. Bollini and J.J. Giambiagi, Physics Letters 40B (1972) 566; J.F. Ashmore, Lettere al Nuovo Cimento 4 (1972) 289.

[36] T. Massam and A. Zichichi, Nuovo Cimento $\underline{43}$ (1966) 1137, and Lettere al Nuovo Cimento 1 (1969) 387.

[37] See also: H.J. Lipkin, in Physique Nucléaire, Les-Houches 1968, ed. C. DeWitt and V. Gillet, Gordon and Breach, N.Y. (1969) 585; H.J. Lipkin, Physics Letters 45B (1973) 267; Y. Nambu, in Preludes in Theoretical Physics, ed. A. de-Shalit et al, North Holland Pub. Comp., Amsterdam (1966) 133.

[38] H. Fritzsch, M. Gell-Mann and H. Leutwyler, Physics Letters 47B (1973) 365. See also: H. Lipkin, Physics Letters 45B (1973) 267. 
[39] O.W. Greenberg, Physical Review Letters 13 (1964) 598.

[40] M.Y. Han and Y. Nambu, Physical Review 139 B (1965) 1006.

[41] G. 't Hooft, Acta Phys. Austr., Suppl. 22 (1980) 531.

[42] T. Massam and A. Zichichi, CERN Report, ISR Users Meeting 10-11 June 1968 (unpublished); M. Basile, G. Cara Romeo, L. Cifarelli, P. Giusti, T. Massam, F. Palmonari, G. Valenti and A. Zichichi, Nuovo Cimento 40A (1977) 41; the complete set of references is reported in this volume.

[43] See: D.J. Gross, in The Rise of the Standard Model, Cambridge University Press (1997) 199; S. Coleman and D.J. Gross, Physical Review Letters 31 (1973) 851.

[44] K. Symanzik, in Proceedings Marseille Conference 19-23 June 1972, ed. C.P. Korthals Altes; id., Lettere al Nuovo Cimento 6 (1973) 77.

[45] S. Coleman, private communication.

[46] D.J. Gross and F. Wilczek, Physical Review Letters 30 (1973) 1343; H.D. Politzer, Physical Review Letters $\underline{30}$ (1973) 1346.

[47] G. 't Hooft, Nuclear Physics B61 (1973) 455.

[48] G. 't Hooft, Nuclear Physics B62 (1973) 444.

[49] S.C. Frautschi, Regge Poles and S-Matrix Theory, W.A. Benjamin Inc. (1963).

[50] V. Gribov, ZhETF 41 (1961) 1962 [SJETP 14 (1962) 1395]; V.N. Gribov and D.V. Volkov, ZhETF 44 (1963) 1068.

[51] H.B. Nielsen and P. Olesen, Nuclear Physics B61 (1973) 45.

[52] B. Zumino, in Renormalization and Invariance in Quantum Field Theory, NATO Adv. Study Institute, Capri, 1973, Ed. R. Caianiello, Plenum (1974) 367.

[53] G. 't Hooft, Nuclear Physics B79 (1974) 276.

[54] A.M. Polyakov, JETP Lett. 20 (1974) 194.

[55] P. Hasenfratz and G. 't Hooft, Physical Review Letters $\underline{36}$ (1976) 1119. 
[56] A.Goldhaber, Physical Review Letters $\underline{36}$ (1976) 1122.

[57] K.G. Wilson, Physical Review D10 (1974) 2445.

[58] L. Onsager, Physical Review 65 (1944) 117; B. Kaufman, Physical Review 76 (1949) 1232; B. Kaufman and L. Onsager, Physical Review 76 (1949) 1244.

[59] M.A. Kramers and G.H. Wannier, Physical Review 60 (1941) 252, 263; L. Kadanoff, Nuovo Cimento 44B (1966) 276.

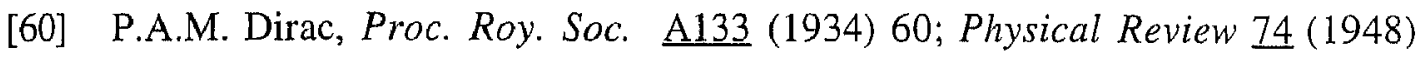
817.

[61] G. 't Hooft, "Gauge Theories with Unified Weak, Electromagnetic and Strong Interactions", in E.P.S. Int. Conf. on High Energy Physics, Palermo, 23-28 June 1975, Editrice Compositori, Bologna 1976, A. Zichichi Ed.

[62] S. Mandelstam, Physics Letters $\underline{\mathrm{B} 53}$ (1975) 476; Physics Reports $\underline{23}$ (1978) 245.

[63] V. Gribov, Nuclear Physics B139 (1978) 1.

[64] G. 't Hooft, Nuclear Physics $\underline{\mathrm{B} 190}$ (1981) 455.

[65] M. Creutz, L. Jacobs and C. Rebbi, Physical Review Letters 42 (1979) 1390.

[66] A.J. van der Sijs, invited talk at the Int. RCNP Workshop on Color Confinements and Hadrons - Confinement 95, March 1995, RCNP Osaka, Japan (hep-th/9505019).

[67] D. Bollini, A. Buhler-Broglin, P. Dalpiaz, T. Massam, F. Navach, F.L. Navarria, M.A. Schneegans and A. Zichichi, Nuovo Cimento 58A (1968) 289 ; the complete set of references is reported in this volume.

[68] A. Zichichi, Proceedings of the 16th International Conference on "High Energy Physics", Batavia, IL, USA, 6-13 Sept. 1972 (NAL, Batavia, 1973), Vol 1, 145.

[69] D. Bollini, A. Buhler-Broglin, P. Dalpiaz, T. Massam, F. Navach, F.L. Navarria, M.A. Schneegans and A. Zichichi, Nuovo Cimento 57A (1968) 404. 
[70] J. Kogut and L. Susskind, Physical Review D9 (1974) 3501, ibid. D10 (1974) 3468, ibid. D11 (1975) 3594.

[71] S. Weinberg, Physical Review D11 (1975) 3583.

[72] A.A. Belavin, A.M. Polyakov, A.S. Schwartz and Y.S. Tyupkin, Physics Letters $\underline{59}(1975) 85$.

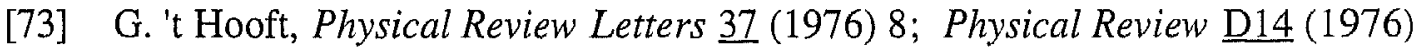
3432; Err. Physical Review D18 (1978) 2199.

[74] R. Jackiw and C. Rebbi, Physical Review D13 (1976) 3398.

[75] R. Jackiw, C. Nohl and C. Rebbi, various Workshops on QCD and solitons, June-Sept. 1977; R. Jackiw and C. Rebbi, Physical Review Letters 37 (1976) 172.

[76] C.G. Callan, R. Dashen and D. Gross, Physics Letters 63B (1976) 334.

[77] R. Crewther, Physics Letters 70B (1977) 359; Rivista del Nuovo Cimento 2 (1979) 63; R. Crewther, in Facts and Prospects of Gauge Theories, Schladming 1978, ed. P. Urban (Springer-Verlag 1978); Acta Phys. Austriaca Suppl. XIX (1978) 47.

[78] G. 't Hooft, Physics Reports 142 (1986) 357.

[79] L. Susskind, private communication.

[80] G. 't Hooft, in Recent Developments in Gauge Theories, Cargèse 1979, ed. G. 't Hooft et al., Plenum Press, New York (1980), Lecture III, "Naturalness, chiral symmetry and spontaneous chiral symmetry breaking", reprinted in: Dynamical Symmetry Breaking, a Collection of reprints, ed. A. Fahri et al., World Scientific, Singapore, Cambridge, (1982) 345.

[81] K. Symanzik, Nuclear Physics B226 (1983) 187, 205; M. Lüscher and P. Weisz, Nuclear Physics B266 (1986) 309; G.P. Lepage and P.B. Mackenzie, Physical Review D48 (1993) 2250, and many more recent publications, see J. Snippe, The Uses of Improved Actions in Lattice Gauge Theory, Leiden thesis, February 19, 1997.

[82] G. 't Hooft, Nuclear Physics B72 (1974) 461. 
[83] T.H. Skyrme, Proc. R. Soc. $\underline{\text { A260 (1961) } 127 .}$

[84] G. 't Hooft, Nuclear Physics B75 (1974) 461.

[85] N. Seiberg, Physics Letters 318B (1993) 469; N. Seiberg and E. Witten, RU94-52, IAS-94-43, hep-th/9407087.

[86] M. Shifman, in Confinement, Duality and Non perturbative Aspects of $Q C D$, P. van Baal ed., Cambridge UK Workshop, June 1997, NATO ASI Series, Plenum Press, New York and London (1998) 477.

[87] Th. Appelquist and H.D. Politzer, Physical Review Letters 34 (1975) 43; Th. Appelquist, A. De Rújula and H.D. Politzer, Physical Review Letters $\underline{34}$ (1975) 365.

[88] G. Sterman and S. Weinberg, Physical Review Letters 39 (1977) 1436.

[89] G. Veneziano, Report in this volume.

[90] M. Basile, G. Cara Romeo, L. Cifarelli, A. Contin, G. D'Alì, P. Di Cesare, B. Esposito, P. Giusti, T. Massam, F. Palmonari, G. Sartorelli, G. Valenti and A. Zichichi, Physics Letters 92B (1980) 367; the complete list of references on the effective energy and universality features is reported in this volume; see also Ref. [89] and the original papers reproduced after Ref. [89].

[91] G. 't Hooft, "The confinement phenomenon in quantum field theory", in 1981 Cargèse Summer School lecture notes on fundamental interactions, eds M. Lévy and J.-L. Basdevant, NATO Adv. Study Inst. Series B: Physics, vol. 85, p. 639.

[92] V.A. Kuzmin, V.A. Rubakov and M.E. Shaposhnikov, Physics Letters B155 (1985) 36.

[93] F.R. Klinkhamer and N.S. Manton, Physical Review D30 (1984) 2212.

[94] G.R. Farrar and M.E. Shaposhnikov, Physics Letters 70 (1993) 2833.

[95] G. 't Hooft, Commun. Math. Phys. $\underline{86}$ (1982) 449; ibid. $\underline{88}$ (1983) 1; G. 't Hooft, "Planar diagram field theories", in Progress in Gauge Field Theory, NATO Adv. Study Inst. Series, eds. G. 't Hooft et al, Plenum (1984) 271. 